\title{
Association of Neutrophil to Lymphocyte Ratio with Lower Patency Rates among Patients with Infrapopliteal Arterial Disease Undergoing Balloon Angioplasty
}

\author{
Abdulkadir Uslu, Regayip Zehır, Elnur Alızade, Ayhan Küp, Zubeyde Bayram, Ahmet Guner, Süleyman Barutcu ${ }^{1}$ \\ Department of Cardiology, Kartal Kosuyolu Heart Research and Training Hospital, Istanbul, 'Department of Cardiology, Van Research and Education Hospital, \\ Van, Turkey
}

\section{Abstract}

Introduction: Percutaneous endovascular methods have emerged to the contemporary revascularization choice in patients with infrapopliteal (IP) arterial disease. However, restenosis remains to be the Achilles' heel of this minimally invasive revascularization techniques. We aimed to analyze the association between preprocedural (neutrophil to lymphocyte ratio) Neutrophil-to-Lymphocyte ratio (NLR) and subsequent patency in a cohort of patients with symptomatic IP disease undergoing balloon angioplasty. Methods: All patients primarily treated with angioplasty of at least one IP artery causing severe symptoms or critical limb ischemia (CLI) (Rutherford category 1-6) between January 2014 and August 2015 were analyzed. The baseline demographic, clinical, and angiographic features admission laboratory test results were obtained from hospital files and computer records. NLR was calculated as the preprocedural ratio of neutrophils to lymphocytes. Results: The study population involved $42(43.8 \%)$ CLI patients and $52(54.2 \%)$ claudicants. The most frequent target vessel was the posterior tibial artery. Primary patency at 1 month was $81.9 \%$ and $62.7 \%$ at 6 months. NLR (odds ratio: $0.04, P=0.03$ ) independently predicted patency at 1 month but did not have a role on arterial patency after 1 month. Only smoking (odds ratio: $4.8, P=0.01$ ) associated with patency at 6 months. Conclusion: Preprocedural NLR was an independent risk factor for IP arterial patency at short-term. It may be used as a risk factor for subsequent amputation or recurrent interventions.

Keywords: Critical limb ischemia, infrapopliteal, neutrophil-to-lymphocyte ratio, patency, percutaneous transluminal angioplasty

\section{INTRODUCTION}

Infrapopliteal (IP) occlusive disease is a severe and diffuse form of peripheral artery disease (PAD) and requires challenging revascularization procedures. Patients suffering from severe lifestyle-limiting claudication or critical limb ischemia (CLI) due to atherosclerosis of the crural arteries are often frailer and have higher amputation and mortality rates. ${ }^{[1]}$ Percutaneous endovascular methods have emerged to the contemporary revascularization choice in these high-risk patients. Production of uninterrupted blood flow to the foot to allow wound healing, alleviation of pain, and preservation of limb mobility are the primary goals of interventions on the IP. ${ }^{[2]}$ The main drawbacks with regard to endovascular interventions of these end arteries were the vessel patency, complications, and technical failure. Because of small caliber and high calcification and occlusion rates, below the knee

\begin{tabular}{|l|l|}
\hline \multicolumn{3}{c|}{ Access this article online } \\
\hline Quick Response Code: & Website: \\
& \\
http://www.ijcva.com
\end{tabular}

arteries is subject to a higher risk of restenosis and thrombosis than the other vascular districts. Percutaneous transluminal angioplasty (PTA) remains a reasonable primary treatment approach for IP despite high re-stenosis and re-intervention rates. Loss of arterial patency after intervention delays ischemic wound healing and achievement of ambulatory status and subsequently hinders improvement of clinical outcome. ${ }^{[3]}$

Neutrophil-to-lymphocyte ratio (NLR), an inflammatory biomarker, related to adverse vascular endpoints in the atherosclerotic spectrum of diseases. ${ }^{[4]}$ It correlates with

Address for correspondence: Dr. Ahmet Guner, Kartal Kosuyolu Heart and Research Hospital, Denizer Street, Kartal, 34846 Istanbul, Turkey. E-mail: ahmetguner488@gmail.com

This is an open access journal, and articles are distributed under the terms of the Creative Commons Attribution-NonCommercial-ShareAlike 4.0 License, which allows others to remix, tweak, and build upon the work non-commercially, as long as appropriate credit is given and the new creations are licensed under the identical terms.

For reprints contact: reprints@medknow.com

How to cite this article: Uslu A, Zehır R, Alızade E, Küp A, Bayram Z, Guner A, et al. Association of neutrophil to lymphocyte ratio with lower patency rates among patients with infrapopliteal arterial disease undergoing balloon angioplasty. Int J Cardiovasc Acad 2018;4:90-5. 
biomarkers of proinflammatory state and reflects both the neutrophilia of inflammation and the relative lymphopenia of the cortisol-induced stress response. Elevated NLR has been associated with a high risk for CLI in PAD patients and higher mortality in patients with PAD who present with CLI. ${ }^{[5,6]}$ NLR also predicts intimal proliferation and resultant restenosis in coronary arteries. ${ }^{[7]}$ However, its predictive role on vessel patency after IP angioplasty in patients with symptomatic IP has not been demonstrated yet. Therefore, we aimed to assess the association between NLR and the 1 and 6 months patency rates in patients with IP after IP intervention. We hypothesized that preprocedural NLR would be associated with patency rate early after intervention but not thereafter.

\section{Methods}

This retrospective observational study was conducted at the Kartal Kosuyolu Heart Training and Research Hospital. All patients primarily treated with PTA of at least one IP artery causing severe symptoms or CLI (Rutherford category 1-6) between January 2014 and August 2015 were analyzed. A total of 134 patients (93 males) of mean age 65 years with 166 limbs were evaluated. Significant stenosis of inflow arteries if present had to be treated successfully before enrollment. The success rate was $93.2 \%$ (125 patients), and technically successful angioplasty of de novo lesions were included. CLI defined as the presence of chronic ischemic rest pain, ulcers, or gangrene attributable to objectively proven IP. ${ }^{[8]}$ Technical success of IP PTA was described as recanalization with the antegrade flow and $30 \%$ or less residual stenosis of below the knee artery. PTA was performed through anterograde (ipsilateral or contralateral) or retrograde approach at the discretion of the treating interventional cardiologist. Stents were implanted if flow-limiting complications occurred. Unless contraindicated, all patients received aspirin, clopidogrel, statin and cilostazol after successful recanalization of the vessel. ON a 1-month course of combination antiplatelet therapy consisting of aspirin (100 mg) and clopidogrel (75 mg) were administered. Patients who failed to take the prescribed medication during follow-up were excluded from the study. Previous peripheral artery bypass graft surgery affecting the target limb, procedural complications requiring further surgical revascularization or immediate amputation postprocedure, amputation of treated limb above the metatarsal level and death of the patient during follow-up and patients with hematological, inflammatory or rheumatological diseases, and those treated with steroids or anti-inflammatory drugs were the exclusion criteria. Fourteen patients underwent amputation, 6 patients died during 6 months of follow-up. Primary patency of vessel attempted was accepted as the endpoint and evaluated at 1 and 6 months. Loss of patency defined as restenosis $(>50 \%)$ or occlusion of the artery intervention on detected by computed tomography angiography or duplex scan. Peak systolic velocity ratio $\geq 2.4$ value calculated on duplex ultrasound has been clinically established to represent $>50 \%$ restenosis. ${ }^{[9]}$ Primary patency was reported as continual patency with no procedure at the treated site or on a segment adjacent to it. The baseline demographic, clinical, and angiographic features admission laboratory test results (including absolute neutrophil and lymphocyte counts) were obtained from hospital files and computer records. Blood samples were collected in calcium-ethylenediaminetetraacetic acid tubes. Blood counts were measured with an auto-analyzer. Neutrophil-to-lymphocyte ratio was calculated as the ratio of neutrophils to lymphocytes in the peripheral blood. Other routine laboratory parameters were also measured in our hospital's laboratory, from the blood samples obtained on the admission of the patients. NLR was calculated as the preprocedural ratio of neutrophils to lymphocytes. C-reactive protein (CRP) levels were measured on Cobas Integra analyzer (Roche Diagnostics, Istanbul, Turkey) using the turbidimetric method. The study was carried out according to the Declaration of Helsinki and was approved by the Local Ethics Committee. Written consent was obtained from all the patients.

Number Cruncher Statistical System (NCSS) 2007 (NCSS, LLC, Kaysville, Utah, USA) program was used for statistical analysis. Continuous variables are presented as mean values + standard deviation, whereas categorical ones are presented as percentage. Student's $t$-test was used for comparing parameters which show normal distribution and Mann-Whitney U-test was used for comparing variables that do not show normal distribution. Pearson Chi-square test was used for comparing categorical variables. Freedom from restenosis and freedom from occlusion based on the time to the first event for each target lesion. Multivariate logistic regression analysis carried out to identify independent predictors of patency. Variables with unadjusted $P<0.1$ by univariate analysis were identified and forced in the full model. Significance was determined at level for $P<0.05$.

\section{RESULTS}

The demographic and clinical characteristics and laboratory results of the study population are summarized in Table 1. There were no significant differences between the two groups in terms of basic clinical features. After exclusions, we identified 94 patients (62 male) who underwent PTA to 144 IP lesions during the study. Study population involved 42 (43.8\%) CLI patients and $52(54.2 \%)$ claudicants. Patients divided into two groups according to patency at 1 month. Primary patency at 1 month was $81.9 \%$ (restenosis in 17 patients), significantly different between the claudicant and CLI cohorts $(P=0.001)$. Clinical characteristics of patients and lesion features with respect to vessel patency at 1 month are summarized in Table 1. Renal failure (glomerular filtration rate $<60 \mathrm{~mL} / \mathrm{min} / 1.73 \mathrm{~m}^{2}$ ) $(P=0.017)$, dialysis $(P=0.001)$ and smoking $(P<0.001)$ were more common in the restenotic group. The most frequent target vessel was the posterior tibial artery in both groups and lesion features did not differ between groups. Preinterventional NLR and CRP levels were significantly higher in the stenotic group. On multivariate analysis CRP (odds: $0.07, P=0.02$ ) and NLR (odds: $0.04, P=0.03$ ) independently predicted patency at 1 month [Table 2]. To evaluate the trend for increase of restenosis rate for increasing NLR and CRP 


\begin{tabular}{|c|c|c|c|}
\hline Characteristics & Infrapopliteal vessel restenosis $(n=17), n(\%)$ & Patent infrapopliteal vessel $(n=77), n(\%)$ & $P$ \\
\hline Age, years & $61.3 \pm 8.1$ & $62.1 \pm 8.6$ & 0.72 \\
\hline Male sex & $14(82.4)$ & $48(62.3)$ & 0.16 \\
\hline Diabetes & $8(47.1)$ & $38(48.4)$ & 0.53 \\
\hline BMI $\left(\mathrm{cm}^{2}\right)$ & $22.1 \pm 2$ & $24.3 \pm 4$ & 0.67 \\
\hline Arrhythmia & $4(23.5)$ & $17(22.1)$ & 0.89 \\
\hline Congestive heart failure & $2(11.8)$ & $19(24.7)$ & 0.24 \\
\hline Coronary artery disease & $5(29.4)$ & $33(42.9)$ & 0.30 \\
\hline CABG or PCI & $5(29.4)$ & $26(33.8)$ & 0.73 \\
\hline Hypertension & $16(94.1)$ & $66(87.5)$ & 0.34 \\
\hline Hyperlipidemia & $4(23.5)$ & $33(42.9)$ & 0.14 \\
\hline Renal insufficiency & $8(47.1)$ & $15(19.5)$ & 0.017 \\
\hline Dialysis & $5(29.4)$ & $3(3.9)$ & 0.001 \\
\hline Stroke & $4(23.5)$ & $9(11.7)$ & 0.20 \\
\hline Current smoker & $14(82.6)$ & $26(33.8)$ & $<0.001$ \\
\hline Medication (cilastazol + statin) & 87 & 83 & $>0.22$ \\
\hline Chronic obstructive pulmonary disease & $3(17.6)$ & $17(22.1)$ & 0.68 \\
\hline \multicolumn{4}{|l|}{ Clinic presentation } \\
\hline Claudicants & $3(17.6)$ & $49(63.9)$ & \multirow[t]{2}{*}{0.001} \\
\hline Critical limb ischemia & $14(82.4)$ & $28(36.4)$ & \\
\hline \multicolumn{4}{|l|}{ Rutherford category } \\
\hline 1 & 0 & $7(9.1)$ & \multirow[t]{6}{*}{0.01} \\
\hline 2 & 0 & $13(16.9)$ & \\
\hline 3 & $3(17.6)$ & $29(30.9)$ & \\
\hline 4 & $4(23.5)$ & $13(13.8)$ & \\
\hline 5 & $10(58.8)$ & $28(29.8)$ & \\
\hline 6 & 0 & $4(4.3)$ & \\
\hline Previous higher level revascularisation & $3(17.6)$ & $29(37.9)$ & 0.11 \\
\hline \multicolumn{4}{|l|}{ Target artery, $n$ (total) } \\
\hline Anterior tibial & $7(27)$ & $31(114)$ & \multirow[t]{4}{*}{0.28} \\
\hline Peroneal & $4(27)$ & $28(114)$ & \\
\hline Posterior tibial & $9(27)$ & $32(114)$ & \\
\hline Tibioperoneal trunk & $7(27)$ & $23(114)$ & \\
\hline Lesion length, $\mathrm{cm}$ & $7 \pm 2.1$ & $6.4 \pm 3$ & 0.51 \\
\hline Baseline stenosis, \% & $79 \pm 13$ & $75 \pm 12$ & 0.21 \\
\hline Total occlusions & $3(17.6)$ & $21(27.3)$ & 0.41 \\
\hline Calcification & $4(23.5)$ & $19(24.7)$ & 0.91 \\
\hline Hemoglobin (mg/dL) & $14 \pm 1.2$ & $13 \pm 1.8$ & 0.13 \\
\hline White blood cells $\left(10^{9} / \mathrm{L}\right)$ & $10 \pm 2$ & $9.8 \pm 1.9$ & 0.44 \\
\hline Fasting glocose (g/dl) & $173 \pm 81$ & $143 \pm 64$ & 0.11 \\
\hline NLR & $6.0 \pm 1.1$ & $2.9 \pm 1$ & $<0.001$ \\
\hline $\mathrm{CRP}, \mathrm{mg} / \mathrm{L}$ & $5.6 \pm 2.2$ & $2.0 \pm 0.8$ & $<0.001$ \\
\hline
\end{tabular}

CABG: Coronary artery bypass graft, CRP: C reactive protein, NLR: Neutrophil-lymphocyte ratio, PCI: Percutaneous coronary intervention, BMI: Body mass index

Jonckheere-Terpstra test was performed and showed statistical significance $(P<0.001$ for both). Seventy-seven patients with patent vessels were followed up until 6 months and patency re-evaluated. Patency at 6 months was $62.7 \%$ (restenosis in 35 patients). 18 patients developed restenosis after the $1^{\text {st }}$ month. Patients divided into two groups according to patency at 6 months. Stent was implanted in 7 patients in the whole patient population. Clinical characteristics of patients and lesion features with respect to vessel patency at 6 months are presented in Table 3. Patients with CLI had also higher restenosis rate at 6 months $(P=0.013)$. There was no difference between groups in vessel diameter, baseline stenosis, the prevalence of chronic occlusion, or extent of lesion calcification. Coronary artery disease $(P=0.02)$, smoking $(P=0.001)$, and CRP levels $(P=0.004)$ were significantly different between groups. On multivariate analysis only smoking (odds ratio: $4.8, P=0.01)$ associated with patency at 6 months [Table 4].

\section{Discussion}

This study allowed evaluation the role of system inflammation on arterial patency rates after successful PTA for IP. The 
findings of the present study show that NLR provides relevant information regarding the risk of restenosis at 1 month.

\begin{tabular}{|c|c|c|}
\hline Parameters & OR (95\% Cl) & $P$ \\
\hline Clinic presentation & $0.73(0.03-17.1)$ & 0.84 \\
\hline Current smoker & $0.31(0.01-9.9)$ & 0.51 \\
\hline Dialysis & $0.02(0-7.4)$ & 0.21 \\
\hline NLR & $0.04(0.002-0.74)$ & 0.03 \\
\hline CRP & $0.07(0.008-0.70)$ & 0.02 \\
\hline
\end{tabular}

Admission NLR was found to be an independent predictor of early restenosis rate.

Restenosis after IP angioplasty is frequent and not fully understood. Preprocedural identification of patients who are likely to benefit from PTA and those who might need recurrent or a more definitive intervention is crucial for the management of IP. The occurrence of restenosis during the wound healing process early after angioplasty would hamper limb salvage. Most of the data on its pathophysiological mechanisms come from the experience on coronary arteries due to their morphological similarities with crural arteries. Barotraumatic vessel dilatation triggers an intense

\begin{tabular}{|c|c|c|c|}
\hline Characteristics & Infrapopliteal vessel restenosis $(n=18), n(\%)$ & Patent infrapopliteal vessel $(n=59), n(\%)$ & $P$ \\
\hline Age, years & $64.3 \pm 10$ & $61.4 \pm 8$ & 0.22 \\
\hline Male sex & $11(61.1)$ & $37(62.7)$ & 0.90 \\
\hline Diabetes & $11(61.1)$ & $27(45.7)$ & 0.25 \\
\hline Arrhythmia & $2(11.1)$ & $15(25.1)$ & 0.20 \\
\hline Congestive heart failure & $5(27.8)$ & $14(23.7)$ & 0.72 \\
\hline Coronary artery disease & $12(66.7)$ & $21(35.6)$ & 0.02 \\
\hline CABG or PCI & $9(50)$ & $17(28.8)$ & 0.09 \\
\hline Hypertension & $15(83.3)$ & $51(86.4)$ & 0.74 \\
\hline Hyperlipidemia & $6(33.3)$ & $27(45.8)$ & 0.35 \\
\hline Renal insufficiency & $5(27.8)$ & $10(16.9)$ & 0.31 \\
\hline Dialysis & $1(5.6)$ & $2(3.4)$ & 0.67 \\
\hline Stroke & $1(5.6)$ & $8(13.6)$ & 0.35 \\
\hline Current smoker & $12(66.7)$ & $14(23.7)$ & 0.001 \\
\hline Medication (cilastazol + statin) & 87 & 83 & $>0.22$ \\
\hline Chronic obstructive pulmonary disease & $4(22.2)$ & $13(22)$ & 0.98 \\
\hline \multicolumn{4}{|l|}{ Clinic presentation } \\
\hline Claudicants & $7(38.9)$ & $42(71.2)$ & 0.013 \\
\hline Critical limb ischemia & $11(61.1)$ & $17(28.8)$ & \\
\hline \multicolumn{4}{|l|}{ Rutherford category } \\
\hline 1 & $3(16.7)$ & $4(6.8)$ & 0.11 \\
\hline 2 & $1(5.6)$ & $12(20.3)$ & \\
\hline 3 & $10(55.6)$ & $16(27.1)$ & \\
\hline 4 & $1(5.6)$ & $8(13.6)$ & \\
\hline 5 & $2(11.1)$ & $16(27.1)$ & \\
\hline 6 & $1(5.6)$ & $3(5.1)$ & \\
\hline Previous higher level revascularization & $7(38.9)$ & $22(37.3)$ & 0.90 \\
\hline \multicolumn{4}{|l|}{ Target artery, $n$ (total) } \\
\hline Anterior tibial & $6(22)$ & $25(91)$ & 0.13 \\
\hline Peroneal & $7(22)$ & $21(91)$ & \\
\hline Posterior tibial & $8(22)$ & $24(91)$ & \\
\hline Tibioperoneal trunk & $1(22)$ & $21(91)$ & \\
\hline Lesion length, $\mathrm{cm}$ & $6.2 \pm 1.7$ & $6.5 \pm 3.3$ & 0.67 \\
\hline Baseline stenosis, $\%$ & $76 \pm 13$ & $75 \pm 12$ & 0.60 \\
\hline Total occlusions & $7(38.9)$ & $14(23.7)$ & 0.20 \\
\hline Calcification & $4(22.2)$ & $15(25.4)$ & 0.78 \\
\hline Hemoglobin (mg/dL) & $13.1 \pm 2$ & $13.3 \pm 1.7$ & 0.69 \\
\hline White blood cells $\left(10^{9} / \mathrm{L}\right)$ & $9.9 \pm 2$ & $9.8 \pm 2$ & 0.79 \\
\hline Fasting glucose (g/dl) & $134 \pm 39$ & $146 \pm 70$ & 0.47 \\
\hline NLR & $3.1 \pm 1.1$ & $2.8 \pm 0.9$ & 0.36 \\
\hline $\mathrm{CRP}, \mathrm{mg} / \mathrm{L}$ & $2.4 \pm 0.6$ & $1.9 \pm 0.8$ & 0.004 \\
\hline
\end{tabular}

CABG: Coronary artery bypass graft, CRP: C reactive protein, NLR: Neutrophil-lymphocyte ratio, PCI: Percutaneous coronary intervention 


$\begin{aligned} & \text { Table 4: The predictors of the patency at } \mathbf{6} \text { months in the } \\
& \text { multivariable logistic regression analyses }\end{aligned}$
\begin{tabular}{lcc} 
Parameters & OR $(\mathbf{9 5} \% \mathbf{C I})$ & $\boldsymbol{P}$ \\
\hline Current smoker & $0.21(0.72-0.059)$ & 0.01 \\
Clinical presentation & $2.4(0.68-8.6)$ & 0.17 \\
Coronary artery disease & $2.9(0.81-10.3)$ & 0.09 \\
CRP & $0.57(0.28-1.1)$ & 0.17 \\
\hline CRP. C reactive protein, OR: Odds ratio, CI: Confidence interval
\end{tabular}

CRP: $\mathrm{C}$ reactive protein, OR: Odds ratio, $\mathrm{CI}$ : Confidence interval

local inflammatory reaction that leads to proliferation and migration of smooth muscle cells, ending up with neointimal thickening and restenosis. ${ }^{[10]}$ Besides this local vascular inflammation, the preexisting systemic inflammatory state also contributes to the restenotic process. The extent of vascular inflammation in response to PTA was associated with restenosis risk. ${ }^{[1]]}$ Violi et al. reported that increased levels of inflammatory markers were associated with poor long-term prognosis in patients with $\mathrm{PAD} .{ }^{[12]}$ The concentrations of inflammatory markers such as CRP, fibrinogen and some cytokines were found to be higher in a nonselective PAD patients population with restenosis. ${ }^{[13]}$ However, it remains indeterminate whether these markers before PTA independently indicates increased risk for restenosis or causally conduce to its occurrence. Neither of them is of clinical utility in this context. NLR and CRP are widely used inflammatory markers reflecting both severity and extent of PAD. ${ }^{[14,15]}$ Higher levels of these markers long after angioplasty indicates lower arterial patency rate and support the thesis that systemic inflammation is involved in the pathogenesis of postangioplasty restenosis. Neutrophil-derived myeloperoxidase promotes atherogenesis and neointimal proliferation. ${ }^{[16]}$ Relatively low lymphocyte count is an expected finding in IP since it is more aggressive atherosclerosis and generates mora pronounced cortisol-induced stress response. Relative lymphopenia was independently associated with higher mortality in CLI. ${ }^{[17]}$ Previously, cardiovascular events have been reported to be associated with inflammatory markers and a less frequent cardiovascular event has been reported in patients with hs-crp levels $<2 \mathrm{mg} / \mathrm{dl}$. ${ }^{[18]}$

NLR accepted as a systemic indicator of vascular atherosclerotic burden. Preprocedural NRL was shown to be a powerful and independent predictor of restenosis in coronary arteries. ${ }^{[19]}$ Spark et al. prospectively analyzed 149 patients with IP and found that admission NLR was significantly associated with limb salvage rates and mortality. ${ }^{[6]}$ Furthermore, NLR provides useful information about postoperative mortality and graft patency after vascular surgery. Recently, Chan et al. re-evaluated role of NLR in patients CLI undergoing PTA in a retrospective fashion and found out that admission NLR was a predictor of clinical outcomes but did not correlate with patency rates at 1 year. ${ }^{[17]}$ They both used the same NLR cut-off value (5.25) for comparison. We also did not find any association between admission NLR and arterial patency beyond 1 month.
Recent studies have emphasized the weight of IP patency toward clinical outcomes of IP patients during long-term follow-up. In a recent study published by Joshi et al. the authors showed that the presence and extent of acute coronary arterial occlusion is associated with exacerbated vascular atherosclerotic inflammation and re-occlusion at 1 month but not afterward. ${ }^{[20]}$ Similarly, Bleda et al. elucidated that CRP values both on admission and at 1 month representing the ongoing inflammatory process had a strong association with 1-year revascularization events, and suggested pre-PTA CRP levels to determine patients at increased risk for poorer outcomes after lower extremity endovascular therapy. ${ }^{[21]}$ Based on the findings of the present study, we thought that vascular inflammatory load might be associated with arterial patency rates only early after a successful intervention. After creating and maintaining straight-line blood flow to the extremities, patient mobility, and limb salvage can occur. Although local inflammatory reaction at vessel wall increases early after the intervention, systemic vascular inflammation would probably subside. None of the inflammatory markers was independently described as risk factor for restenosis, 6 months after femoral PTA. Similarly, successful lower extremity angioplasty with the reestablishment of peripheral arterial perfusion improves systemic vascular endothelial functions and reduces inflammatory cell counts at 1 month of follow-up. ${ }^{[22]}$ We adopted strict exclusion criteria to avoid possible confounding factors capable of increasing systemic inflammatory status such as failure of limb salvage after the first attempt, amputation or re-intervention. Consistent with previous studies, patency rate at 6 months was not related to baseline systemic inflammatory burden in our cohort. We identified only smoking as an independent predictor of restenosis at 6 months. Smoking is the major risk factor associated with the development and progression of the peripheral arterial disease and vascular inflammatory reaction induced by smoking may contribute to restenosis process. ${ }^{[23]}$

Our finding that periprocedural NLR predicts early loss of primary patency also raises the question of whether anti-restenosis concepts targeting systemic vascular inflammatory load before the time of intervention could improve vessel patency after angioplasty.

Some limitations of this study should be taken into account before reviewing our results. This study represents single-center experience and lack long-term follow-up data. Furthermore, the retrospective nature of the investigation is an inherited weakness. Angiographically verified restenosis of IP arteries was shown to correlate with IP lesion length in the literature. Mean lesion length was similar to previous articles and was $6.5 \mathrm{~cm}$ in this study. ${ }^{[24]}$ However, we did not identify any lesion characteristics as independent predictors of short and mid-term. Exclusion criteria, relatively short follow-up time and design of our investigation may help to explain this difference. In addition, our study is a cross-sectional study and studies with more patient populations are required. 
Elevated NLR at the time of IP balloon angioplasty is associated with significantly decreased arterial patency at 1 month. Supporting the hypothesis that vascular inflammation is a key factor in the restenotic process, we identified preprocedural NLR as an independent risk factor for IP arterial patency at short-term. Like other studies, inflammatory markers did not correlate with restenosis on crural arterial territory mid-term. Hence, preprocedural NLR may also be a risk factor for subsequent amputation or need for surgical revascularization early after PTA among patients with IP.

\section{Financial support and sponsorship}

Nil.

\section{Conflicts of interest}

There are no conflicts of interest.

\section{RefEREnCES}

1. Rand T, Uberoi R. Current status of interventional radiology treatment of infrapopliteal arterial disease. Cardiovasc Intervent Radiol 2013;36:588-98.

2. Sigala F, Kontis E, Hepp W, Filis K, Melissas J, Mirilas P, et al. Long-term outcomes following 282 consecutive cases of infrapopliteal PTA and association of risk factors with primary patency and limb salvage. Vasc Endovascular Surg 2012;46:123-30.

3. Iida O, Soga Y, Kawasaki D, Hirano K, Yamaoka T, Suzuki K, et al. Angiographic restenosis and its clinical impact after infrapopliteal angioplasty. Eur J Vasc Endovasc Surg 2012;44:425-31.

4. Verdoia M, Barbieri L, Di Giovine G, Marino P, Suryapranata H, De Luca G, et al. Neutrophil to lymphocyte ratio and the extent of coronary artery disease: Results from a large cohort study. Angiology 2016;67:75-82.

5. Gary T, Pichler M, Belaj K, Hafner F, Gerger A, Froehlich H, et al. Neutrophil-to-lymphocyte ratio and its association with critical limb ischemia in PAOD patients. PLoS One 2013;8:e56745.

6. Spark JI, Sarveswaran J, Blest N, Charalabidis P, Asthana S. An elevated neutrophil-lymphocyte ratio independently predicts mortality in chronic critical limb ischemia. J Vasc Surg 2010;52:632-6.

7. Balli M, Taşolar H, Çetin M, Tekin K, Çă̆liyan ÇE, Türkmen S, et al. Use of the neutrophil to lymphocyte ratio for prediction of in-stent restenosis in bifurcation lesions. Eur Rev Med Pharmacol Sci 2015;19:1866-73.

8. Elsayed S, Clavijo LC. Critical limb ischemia. Cardiol Clin 2015;33:37-47.

9. Ranke C, Rieder M, Creutzig A, Alexander K. A nomogram of duplex ultrasound quantification of peripheral arterial stenoses. Studies of the cardiovascular model and in angiography patients. Med Klin (Munich) 1995;90:72-7.

10. Tiroch KA, Byrne RA, Kastrati A. Pharmacological prevention and management of restenosis. Expert Opin Pharmacother 2010;11:1855-72.

11. Joviliano EE, Piccinato CE, Dellalibera-Joviliano R, Moriya T, Évora $\mathrm{PR}$. Inflammatory markers and restenosis in peripheral percutaneous angioplasty with intravascular stenting: Current concepts. Ann Vasc Surg 2011;25:846-55.

12. Violi F, Criqui M, Longoni A, Castiglioni C. Relation between risk factors and cardiovascular complications in patients with peripheral vascular disease. Results from the A.D.E.P. study. Atherosclerosis 1996; $120: 25-35$

13. Kotschy D, Kotschy M, Masłowski L, Socha P, Kwapisz J, Czyżewska-Buczyńska A, et al. Inflammatory markers in peripheral arterial diseases patients after endovascular revascularization with new restenosis. Acta Angiol 2014;20:47-59.

14. Vainas T, Stassen FR, de Graaf R, Twiss EL, Herngreen SB, Welten RJ, et al. C-reactive protein in peripheral arterial disease: Relation to severity of the disease and to future cardiovascular events. J Vasc Surg 2005;42:243-51

15. Amrock SM, Weitzman M. Multiple biomarkers for mortality prediction in peripheral arterial disease. Vasc Med 2016;21:105-12.

16. Tiyerili V, Camara B, Becher MU, Schrickel JW, Lütjohann D, Mollenhauer M, et al. Neutrophil-derived myeloperoxidase promotes atherogenesis and neointima formation in mice. Int $\mathbf{J}$ Cardiol 2016;204:29-36

17. Chan C,Puckridge P,Ullah S, Delaney C, SparkJI.Neutrophil-lymphocyte ratio as a prognostic marker of outcome in infrapopliteal percutaneous interventions for critical limb ischemia. J Vasc Surg 2014;60:661-8.

18. Lorenzatti A, Servato ML. Role of anti-inflammatory interventions in coronary artery disease: Understanding the canakinumab anti-inflammatory thrombosis outcomes study. Eur Cardiol 2018;13:38-41.

19. Turak O, Ozcan F, Isleyen A, Tok D, Sokmen E, Buyukkaya E, et al. Usefulness of the neutrophil-to-lymphocyte ratio to predict bare-metal stent restenosis. Am J Cardiol 2012;110:1405-10.

20. Joshi NV, Toor I, Shah AS, Carruthers K, Vesey AT, Alam SR, et al. Systemic atherosclerotic inflammation following acute myocardial infarction: Myocardial infarction begets myocardial infarction. J Am Heart Assoc 2015;4:e001956.

21. Bleda S, de Haro J, Varela C, Acin F. C-reactive protein and endovascular treatment of lower limb peripheral artery disease: An independent prognostic factor. J Endovasc Ther 2015;22:233-9.

22. Husmann M, Dörffler-Melly J, Kalka C, Diehm N, Baumgartner I, Silvestro A, et al. Successful lower extremity angioplasty improves brachial artery flow-mediated dilation in patients with peripheral arterial disease. J Vasc Surg 2008;48:1211-6.

23. Schillinger M, Exner M, Mlekusch W, Haumer M, Sabeti S, Ahmadi R, et al. Effect of smoking on restenosis during the $1^{\text {st }}$ year after lower-limb endovascular interventions. Radiology 2004;231:831-8.

24. Baumann F, Willenberg T, Do DD, Keo HH, Baumgartner I, Diehm N, et al. Endovascular revascularization of below-the-knee arteries: Prospective short-term angiographic and clinical follow-up. J Vasc Interv Radiol 2011;22:1665-73. 